\title{
Vocal cord paralysis secondary to anabolic steroid abuse
}

Keywords: vocal cord paralysis, hiv, hepatic dysfunction, cholestatic jaundice, azoospermia, hepatic adenomas, laryngitis, hypertrophy

\section{Introduction}

Testosterone was first isolated in 1935. Since then, numerous synthetic derivatives of the hormone, which are collectively termed anabolic-androgenic steroids (AAS), have been produced and are increasingly used in both medical and non-medical settings. ${ }^{2,3}$ The androgenic and anabolic effects of such agents have been shown to enhance muscle mass and strength as well as creating a stimulatory effect on androgen receptors in the brain causing increased euphoria, aggression, and improved endurance. ${ }^{4,5}$ Medicinal uses of AAS include treatment of anaplastic anaemia as well as promoting muscle depositing in age-related sarcopaenia and weight gain following major burns. ${ }^{6-8}$ More recently AAS have shown to play a role in the treatment of muscle wasting secondary to human immunodeficiency virus (HIV). ${ }^{9}$

It is these muscle stimulating properties of AAS that have lead to their common use amongst athletes and bodybuilders. ${ }^{10}$ Studies have shown the prevalence of AAS use in adolescents to be $3.6 \%$. However, amongst athletes prevalence increases to $22.7 \%{ }^{11,12}$ Often, such groups exceed supraphysiological requirements leading to toxic side effects of AAS. The prevalence of such reactions is difficult to ascertain due to under reporting in this patient population. ${ }^{13}$ However, common side effects of AAS are known to include hepatic dysfunction, cholestatic jaundice, azoospermia, hepatic adenomas, and dependence..$^{14,15}$

Several studies have examined the impact of AAS on the larynx. Known changes include laryngeal enlargement and thickening of the vocal cords (VC) leading to a lower pitched voice. ${ }^{16,17}$ Severe laryngitis has also been shown to be a potential side effect of AAS. ${ }^{18}$ However, we can find no literature on the relationship between AAS and $\mathrm{VC}$ or vocal nerve palsy. In light of the lack of literature on this subject, we report the first case of stridor and vocal cord palsy in a chronic anabolic steroid user.

\section{Case report}

A 37year -old male, who identified as a 'body builder,' was admitted under the acute medical assessment unit with complaints of sudden-onset shortness of breath, which was constant in nature and associated with 'tightness' in the throat. On examination he was afebrile but had laboured breathing. He was also noted to have a hoarse voice. These symptoms were sudden onset in nature and had been present for several hours prior to presentation. Oxygen saturation was $100 \%$ on room air while respiratory and heart rate were $25 / \mathrm{min}$ and $76 / \mathrm{min}$, respectively. Neurological and abdominal examinations were unremarkable. Complete blood count, arterial blood gases, electrocardiogram, and chest x-ray were all deemed to be normal. History revealed that the patient regularly took the anabolic steroid Deca DurabolinÒ (nandrolone decanoate) and had done so
Volume 5 Issue I - 2016

\author{
Muhammad Shakeel, PF McLean, A Jawad, C \\ Brewis \\ Department of Otolaryngology head and neck surgery \\ Aberdeen Royal Infirmary, UK
}

\author{
Correspondence: Muhammad Shakeel Department of \\ Otolaryngology head and neck surgery Ward 210 Aberdeen \\ Royal Infirmary Aberdeen AB25 2ZN, UK, \\ Email drshakeel@doctors.org.uk \\ Received: September 04, 2016 | Published: December 07, \\ 2016
}

for four years prior to admission. The patient admitted to injecting AAS once per week. The patient denied taking any other medications. Interestingly, the patient reported that he had experienced similar symptoms two days earlier and attended his primary care physician for advice. He was prescribed a salbutamol inhaler but reported that this offered minimal relief of his symptoms. Additionally, he had experienced similar symptoms intermittently whilst working out at the gym. A provisional diagnosis of an allergic reaction was made. The patient improved with administration of chlorphenamine and was discharged.

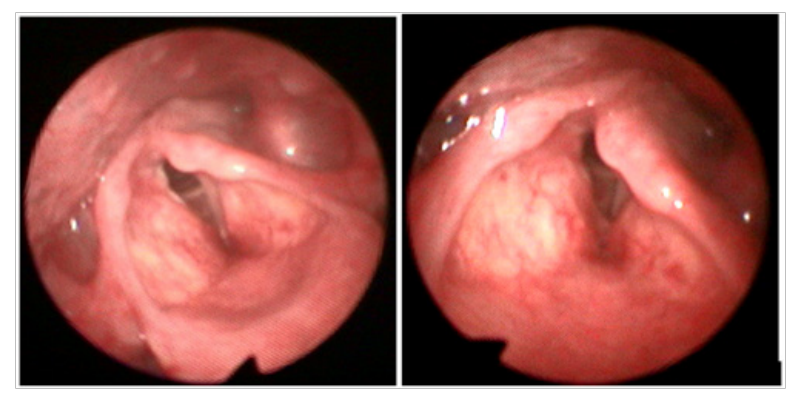

Figure I Left vocal cord palsy as seen on transnasal laryngoscopy-the left $\mathrm{VC}$ is in paramedian position with anteroposterior shortening of the left aryepiglottic fold.

Ten days later, he was admitted to the ear, nose, throat (ENT) emergency clinic with worsening of ongoing symptoms, which were characterised by deteriorating breathlessness on minimal exertion. Subjectively, breathy and hoarse voices were noted. More concerning, marked stridor was also present. Salbutamol, antihistamines, prednisolone and amoxicillin offered minimal relief.

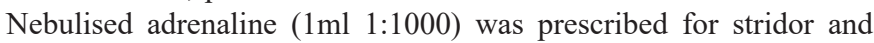
further examination was performed by means of nasal endoscopy 
which showed left VC palsy (Figure 1). Subsequently, a computerised tomography scan of his neck and chest was performed which showed no superior mediastinal mass or neck soft tissue mass. No other cause of vocal cord palsy was identified. He significantly improved the next morning and upon discharge, he was strongly advised against the use of anabolic steroids and was informed about his underlying problem.

Three months later, he was seen in the otolaryngology outpatient clinic where he confirmed avoiding taking AAS. His breathing had significantly improved and his voice was almost back to normal He denied any other ongoing throat symptoms. He was not keen for reassessment of his vocal cords by flexible laryngoscopy. He was discharged from the clinic.

\section{Discussion}

Steroid abuse, that is consuming supraphysiological amounts of anabolic androgenic steroids (AAS) for non-medical purposes, poses a significant public health problem with more than three million Americans currently misusing AAS. Anabolic steroids remain the most common pharmacological agent detected by anti-doping tests in the summer Olympic Games. ${ }^{19}$ Indeed, the last 10years have seen an increased prevalence of AAS, particularly among adolescents and athletes. ${ }^{3}$ However, it is thought that most AAS abusers today are non-elite sportsmen (recreational gym attendees) using the drugs for cosmetic purposes. Evidence is now emerging regarding the increasing problem of muscle dysmorphia or so called 'bigoreixa' in males. ${ }^{20}$ Muscle dysmoprhia is defined as a pre-occupation with increasing muscles mass or leanness. This body-related anxiety often results in men turning to AAS to achieve their perceived goals.

In the United Kingdom, AAS are controlled as Class C substances under the Misuse of Drugs Act 1971. Consequently, AAS are often acquired from unreliable sources and the precise steroid agent and associated side effects are often unknown prior to consumption. It has been reported that AAS misusers can consume 10 to 100 times the recommended therapeutic dose of steroid leading to significant adverse effects. ${ }^{21}$ Reported side effects of AAS misuse include increased sexual drive, development of acne, increase in body hair, and heightened aggression. Additionally, testicular atrophy and a decrease in sperm quality and quantity may occur secondary to suppression of gonadotropin production. Furthermore, AAS are known to be a risk factor for cardiovascular complications including myocardial infarction, development of cardiomyopathy, and sudden cardiac death. ${ }^{14}$

The most common otolaryngology complication secondary to AAS is alteration in the voice. In patients receiving regular anabolic steroids for medical use, a change in voice can be seen in up to $10 \%{ }^{22} \mathrm{~A}$ 'safe dose' has not yet been reported in order to avoid such symptoms. ${ }^{23}$ The most noticeable change is a lowering in frequency of the voice as well a marked lowering in pitch. ${ }^{24}$ Talaat et al., ${ }^{22}$ examined histological changes in the larynx of female mice that had been treated with AAS for up to three months. Reversible changes included vascular congestion, oedema and perivascular and periductal ceullular infiltration. ${ }^{22}$ Irreversible changes included squamous metaplasia and parakeratosis of the epithelium and hypertrophy of the muscle fibers of the thyroarytenoid muscle..$^{22}$ At the time of this writing, no reports could be found of structural or histological laryngeal changes in males.

In our case, we present a sudden onset vocal cord palsy in a 37 year-old man who admitted to chronic anabolic steroid misuse. Vocal cord palsy is a relatively common clinical occurrence seen in $0.42 \%$ of the population. ${ }^{25}$ The vocal cords are innervated by the recurrent laryngeal nerves, which can be damaged secondary to local malignancy, surgery, intubation, or idiopathic causes ${ }^{26}$ Gerritsma et al., ${ }^{23}$ examined virilisation of the voice in post-menopausal woman taking AAS and suggested that changes in the rigidity of the vocal folds secondary to an increase in muscle deposition may be the cause of the lower vocal frequency commonly seen. ${ }^{23}$ One could hypothesize that increased rigidity could ultimately lead to vocal cord paralysis given the extremely high amounts of AAS taken. Alternatively, vocal cord paralysis could occur due to damage to the recurrent laryngeal nerves. Studies have demonstrated AAS to be neurotoxic when injected in the proximity of peripheral nerves. ${ }^{27}$ Although the exact mechanism by which this occurs is poorly understood, it is postulated that AAS initiate the apoptotic cascade of neuronal cells. ${ }^{28}$

\section{Conclusion}

AAS misuse is common and associated with a plethora of adverse effects. Due to the illicit nature of AAS, evidence of such side effects often go undocumented and are limited to case reports. Given the scale of AAS it is important that interesting complications of these pharmacological agents are reported. This case report serves to demonstrate the need for clinicians to consider AAS in the differential of a young, athletic male presenting to the ER or ENT clinic without a clear etiology for vocal cord palsy.

\section{Acknowledgments}

None.

\section{Conflicts of interest}

Author declares there are no conflicts of interest.

\section{Funding}

None.

\section{References}

1. Freeman ER, Bloom DA, McGuire EJ. A brief history of testosterone. $J$ Urol. 2001;165(2):371-373.

2. McCabe SE, Brower KJ, West BT, et al. Trends in non-medical use of anabolic steroids by U.S. college students: results from four national surveys. Drug Alcohol Depend. 2007;90(2-3):243-251.

3. Johnston LD, O'Malley PM, Bachman JG, et al. Monitoring the Future: National Survey Results on Drug Use, 1975-2009. Volume I: Secondary School Students. NIH Publication, Natl Inst Drug Abus. 2010. p.10-7584.

4. Cohen J, Collins R, Darkes J, et al. A league of their own: demographics, motivations and patterns of use of 1,955 male adult non-medical anabolic steroid users in the United States. Journal of the International Society of Sports Nutrition. 2007;4:12.

5. Shahidi N. A review of the chemistry, biological action, and clinical applications of anabolic-androgenic steroids. Clin Ther. 2001;23(9):1355-1390.

6. Gardner FH. Anabolic steroids in aplastic anemia. Acta Endocrinol Suppl (Copenh). 1985;271:87-96.

7. Bhasin S, Tenover JS. Age-Associated Sarcopenia-Issues in the Use of Testosterone as an Anabolic Agent in Older Men. The Journal of Clinical Endocrinology \& Metabolism. 2013;82(6):1659-1660.

8. Robert DH, Leslie DRN. Oxandrolone, an Anabolic Steroid, Significantly Increases the Rate of Weight Gain in the Recovery Phase after Major Burns. Journal of Trauma and Acute Care Surgery. 1997;43(1):47-51. 
9. Orr R, Fiatarone Singh M. The Anabolic Androgenic Steroid Oxandrolone in the Treatment of Wasting and Catabolic Disorders: a review of efficacy and safety. Drugs. 2004;64(7):725-750.

10. Wilson JD. Androgen abuse by athletes. Endocr Rev. 1988;9(2):181-199.

11. Nilsson S, Baigi A. The prevalence of the use of androgenic anabolic steroids by adolescents in a county of Sweden. Eur J Public Health. 2001;11(2):195-197.

12. Alsaeed I, Alabkal J. Usage and perceptions of anabolic-androgenic steroids among male fitness centre attendees in Kuwait-a cross-sectiona study. Substance Abuse Treatment Prevention and Policy. 2015;10:33.

13. Sjöqvist F, Garle M, Rane A. Use of doping agents, particularly anabolic steroids, in sports and society. Lancet. 2008;371(9627):1872-1882.

14. Hartgens F, Kuipers H. Effects of androgenic-anabolic steroids in athletes. Sport Med. 2004;34(8):513-554.

15. Maravelias C, Dona A, Stefanidou M, et al. Adverse effects of anabolic steroids in athletes. A constant threat. Toxicol Lett. 2005;158(3):167-175.

16. Wardle PG, Whitehead MI, Mills RP. Non-reversible and wide ranging voice changes after treatment with danazol. $\mathrm{Br}$ Med J (Clin Res Ed). 1983;287(6397):946.

17. Hall RC, Hall RC. Abuse of supraphysiologic doses of anabolic steroids. South Med J. 2005;98(5):550-555.

18. Ray S, Masood A, Pickles J, et al. Severe laryngitis following chronic anabolic steroid abuse. J Laryngol Otol. 2008;122(3):230-232.

19. Fitch KD. Androgenic-anabolic steroids and the Olympic Games. Asian J Androl. 2008;10(3):384-390.
20. Mosley PE. Bigorexia: bodybuilding and muscle dysmorphia. Eur Eat Disord Rev. 2009;17(3):191-198.

21. DrugFacts: Anabolic Steroids. What are anabolic steroids? National Institute on Drug Abuse (NIDA), USA. 2015

22. Talaat M, Talaat AM, Kelada I, et al. Histologic and histochemical study of effects of anabolic steroids on the female larynx. Ann Otol Rhinol Laryngol. 1987;96(4):468-471.

23. Gerritsma EJ, Brocaar MP, Hakkesteegt MM, et al. Virilization of the voice in post-menopausal women due to the anabolic steroid nandrolone decanoate (Decadurabolin). The effect of medication for one year. Clin Otolaryngol Allied Sci. 1994;19(1):79-84.

24. Damrose EJ (2009) Quantifying the impact of androgen therapy on the female larynx. Auris Nasus Larynx 36(1): 110-112.

25. Ahmad S, Muzamil A, Lateef M. A Study of incidence and etiopathology of vocal cord paralysis. Indian J Otolaryngol Head Neck Surg. 2002;54(4):294-296.

26. Lee M, Lilien DL, Ramaswamy MR, et al. Unilateral Vocal Cord Paralysis Causes Contralateral False-Positive Positron Emission Tomography Scans of the Larynx. Ann Otol Rhinol Laryngol. 2005;114(3):202-206.

27. Dickerman RD, Schaller F, McConathy WJ. Left Ventricular Wall Thickening Does Occur in Elite Power Athletes with or without Anabolic Steroid Use. Cardiology. 1998;90(2):145-148.

28. Estrada M, Varshney A, Ehrlich BE. Elevated testosterone induces apoptosis in neuronal cells. $J$ Biol Chem . 2006;281(35):25492-25501. 